\title{
Redes e intercambio de capitales en condiciones de pobreza: dimensión relacional y dimensión vincular
}

\author{
Alicia B. Gutiérrez- CONICET-UNC-CSE
}

\section{Resumen}

Este trabajo refiere al estudio de las redes sociales implicadas en las estrategias de reproducción social de familias pobres, implicando tanto discusiones teóricas como análisis empíricos.

En primer lugar, explicitaré los conceptos de red y de capital social que utilizo en mis investigaciones, haciendo hincapié en el modo como se insertan en una problemática más amplia: la de las estrategias de reproducción social en la pobreza, en el marco de un análisis estructural.

En segundo lugar, mencionaré distintos tipos de redes construidas empíricamente, que permiten visualizar diferentes intercambios de capitales. Presento aquí el análisis concreto de una de ellas, que articula a un grupo de familias residentes en un barrio pobre cordobés con otros agentes (un grupo de militantes Montoneros) que ocupan otras posiciones en el espacio social.

Finalmente, propongo una doble dimensión analítica para dar cuenta de las redes sociales: una dimensión relacional que permite observar las condiciones materiales y simbólicas, externas e incorporadas, que las hacen posible, y otra vincular, que permite abordar concretamente la frecuencia e intensidad de las interacciones y los tipos de bienes y servicios que circulan en ellas.

Palabras clave: redes, capital social, estrategias de reproducción, dimensión relacional, dimensión vincular

\begin{abstract}
This paper refers to the study of the social networks involved in the strategies of social reproduction of poor families, in the view of theoretical discussions as well as empirical analysis.

In the first place I explain the concept of network and social capital that I utilize in my investigations, emphasizing the way in which they insert themselves within a wider problematic: that of the strategies of social reproduction in poverty, within the framework of a structural analysis.

In the second place, I mention the different types of networks built empirically, that allow visualizing distinct interchanges of capitals. Here I present a concrete analysis of one of them that articulates a group of families living in a poor neighborhood of Cordoba with other agents (a group of militants of Montoneros) that occupy another position in the social space.

Finally, I propose a double analytical dimension to study the social networks: a relational dimension that allows to observe the material and symbolic conditions, outsiders and incorporated, that make them possible and another one, a bonding condition, that permits to attack concretely the frequency and intensity of interactions and the types of goods and services that circulate among them.
\end{abstract}

Key words: networks, social capital, reproduction strategies, relational dimension, bonding dimension

\footnotetext{
${ }^{1}$ Enviar correspondencia a: Alicia Gutiérrez, aliciagutierrez@arnet.com.ar
} 
REDES- Revista hispana para el análisis de redes sociales

Vol. 14,\#4, Junio 2008

http: // revista-redes. rediris.es

\section{El capital social: recurso y relaciones, desde una perspectiva relacional}

Al plantear el análisis relacional en los estudios de la pobreza, se mencionan siempre dos conceptos en primera instancia: redes y capital social, conceptos que ya tienen una cierta tradición en el debate de las ciencias sociales, aunque sus diferentes construcciones remiten a perspectivas también diferentes de la acción social. Quiero referirme brevemente a ellos para mostrar en qué sentido es relacional la dimensión analítica que explicito aquí, tomando en primer lugar el de capital social ${ }^{2}$.

Woolcock y Narayan (2000) reconstruyen su larga historia intelectual en las ciencias sociales $y$, desconociendo completamente la conceptualización de Bourdieu, concluyen que:

“(...) las investigaciones fundacionales de Coleman (1987, 1988 y 1990) en el campo de la educación y de Putnam (1993 y 1995) sobre la participación cívica

y el comportamiento de las instituciones son la fuente de inspiración de la mayor parte de los estudios actuales" (Woolcock y Narayan, op. cit.: 226).

Esos trabajos son agrupados según cuatro "visiones" diferentes del problema: la visión comunitaria, la visión de redes, la visión institucional y la visión sinérgica (I bíd. ).

Millán y Gordon reconstruyen también los aspectos fundamentales del problema, haciendo hincapié en las tres perspectivas que, a su juicio, realizaron los principales aportes a la problemática:

“James Coleman, porque es el clásico de la formulación del concepto; Robert Putnam por la indiscutible influencia de su propuesta sobre capital social y compromiso cívico; y Nan Lin por sus aportaciones a la perspectiva de redes, enmarcadas en el capital social, que se ha constituido en una fuerte corriente de análisis empírico" (Millán y Gordon, 2004: 713).

\footnotetext{
${ }^{2}$ Son numerosos los trabajos que reconstruyen los diferentes aspectos de su historia, y el debate que ha generado desde distintas perspectivas analíticas. En Gutiérrez (2007), tomo la noción de "capital social", teniendo en cuenta la importancia que ha cobrado particularmente en los análisis de situaciones de pobreza, mostrando que sus diferentes conceptualizaciones remiten a teorías de la acción diferentes ( $y$, en algunos casos opuestas) y por ello, proponen construcciones diferentes del problema y, con ello, posibilidades de generar estrategias de abordaje (en el plano analítico y en el político) también diferentes.
} 


\section{REDES- Revista hispana para el análisis de redes sociales Vol. 14,\#4, Junio 2008 \\ http: // revista-redes. rediris.es}

En el contexto argentino, Hintze (2004) realiza una interesante reconstrucción en torno a los aspectos fundamentales del concepto de capital social según distintas vertientes teóricas (y sobre todo en relación con sus límites y debilidades) y de sus vínculos con el concepto de estrategias de supervivencia y, con ello, con los análisis de la pobreza. La autora expresa que Bourdieu y Coleman,

“(con las diferencias que existen entre ambos) sostienen una perspectiva 'estructural' del capital social en contraposición de la 'disposicional o cultural', tal como lo conciben Putman o Fukuyama" (Hintze, 2004: 150).

En efecto, tanto Coleman (1990) como Bourdieu (1980) asocian las nociones de red y de capital social, y, con ello, quedan ligadas más bien a la "estructura" que a la "subjetividad". Ahora bien, me interesa señalar también, siguiendo a Baranger (2000), profundas diferencias entre ambos enfoques.

En primer lugar, Coleman se refiere a un enfoque interaccionista del problema (las redes se explican a través de las interacciones concretas y reales entre individuos) frente a I perspectiva de Bourdieu, que privilegia el análisis de las estructuras que dan fundamento a las interacciones y a las conductas individuales. De todas maneras, por su misma postura de que los agentes no son meros autómatas totalmente determinados por las estructuras, dicho análisis también tiene en cuenta el nivel de las prácticas concretas y de las interacciones.

En segundo lugar, Coleman le da un contenido sustancialista a la noción de capital en general:

"se trata en definitiva de una 'cosa', en el sentido de algo que se crea y está allí, independientemente de cuál pueda ser la modalidad de su utilización (...) mientras el capital físico es totalmente tangible, y el humano ya lo es menos por estar incorporado en las habilidades y el conocimiento adquiridos por un individuo, el capital social es aún menos tangible, al estar incorporado en las relaciones entre las personas" (Baranger, 2000: 11)

Frente a esta noción sustancialista de capital, la que Bourdieu propone remite claramente a Marx y sostiene que el capital, antes que una cosa, es una relación social. 
REDES- Revista hispana para el análisis de redes sociales

Vol. 14,\#4, Junio 2008

http: // revista-redes. rediris.es

Finalmente, señala Baranger, en el concepto estrechamente económico de Coleman no está en absoluto presente la idea de dominación, en oposición a la concepción de Bourdieu, que, remitiendo a la visión marxiana, define a las distintas especies de capital como diferentes especies de poder que se distribuyen desigualmente en los distintos campos, generando con ello estructuras de posiciones de dominacióndependencia.

En definitiva, al concepto sustancialista de capital que plantea Coleman (1988), Bourdieu le opone una concepción del capital que es fundamentalmente relacional en todas sus especies y sus sub-especies, incluyendo, por supuesto, el capital social.

Ahora bien, coincido con Baranger en el sentido en que este capital social no deja de ser relacional en otro aspecto que es fundamental para lo que pretendo plantear en este trabajo: es también relacional en la medida en que aparece basándose primariamente en otro tipo de relaciones, que son justamente las relaciones que toma en cuenta el análisis de redes sociales. Así, el capital social viene a ser relacional por partida doble, al estar referido a relaciones sociales entre agentes que interactúan (Baranger, 2000) ${ }^{3}$.

En el marco global de la perspectiva analítica relacional que propongo para el estudio de la pobreza4, sugiero entonces considerar el capital social (en relación con la noción de red social) como:

“conjunto de recursos actuales o potenciales que están ligados a la posesión de una red duradera de relaciones más o menos institucionalizadas de interconocimiento y de inter-reconocimiento; o, en otros términos, a la pertenencia a un grupo, como conjunto de agentes que no están solamente

\footnotetext{
3 En Gutiérrez, 2005, retomo en detalle estos aspectos, pretendiendo demostrar las capacidades heurísticas del concepto de capital social en Bourdieu. Inspirada en esta idea de Baranger y sobre la base de un estudio empírico, explicito diferentes dimensiones que dan cuenta de lo "relacional" del capital social (es un recurso, que puede tomar diferentes formas, que posiciona a los agentes sociales en estructuras de poder, que permite relacionar a los pobres entre sí y a los pobres con no-pobres, que es también relacional en función de la estructura en un doble sentido y que constituye una apuesta -enjeu- en un doble sentido).

4 El concepto central de esta perspectiva teórica es el de "estrategias de reproducción social", cuyos factores explicativos son: volumen y estructura del capital (capital económico, cultural, social y simbólico) que hay que reproducir; instrumentos de reproducción social; estado de la relación de fuerzas entre las clases; y habitus incorporados. Se trata de un concepto que posibilita explicar y comprender las estrategias que ponen en marcha las diferentes familias insertas en posiciones de clase diferentes (o de fracciones de clase diferentes), partiendo del supuesto de que todas esas estrategias se entrelazan en un marco explicativo-comprensivo relacional. (Gutiérrez, 2004a y 2006). Para el caso de las estrategias de familias pobres, ello implica, por lo tanto, que sus estrategias son relacionales respecto a las de las familias no-pobres; es decir, en definitiva, que la pobreza no se reproduce independientemente de la riqueza. Éste es sólo uno de los aspectos - aunque a los efectos de este trabajo el fundamental- de las dimensiones teóricas y metodológicas implicadas en esa perspectiva relacional.
} 


\section{REDES- Revista hispana para el análisis de redes sociales Vol. 14,\#4, Junio 2008 \\ http: // revista-redes. rediris.es}

dotados de propiedades comunes (susceptibles de ser percibidas por el observador, por los otros o por ellos mismos) sino que están también unidos por lazos permanentes y útiles" (Bourdieu, 1980: 2 -subrayado del autor-).

El capital social está ligado a un círculo de relaciones estables que son el producto de:

"estrategias de inversión social consciente o inconscientemente orientadas hacia la institución o reproducción de relaciones sociales directamente utilizables, a corto o a largo plazo" (I bídem).

En otras palabras, sería el conjunto de relaciones sociales que un agente puede movilizar en un momento determinado, que le pueden proporcionar un mayor rendimiento del resto de su patrimonio (los demás capitales, económico y cultural especialmente). El capital social es, por otra parte, como todo capital, un poder que exige inversiones permanentes, en tiempo, en esfuerzo, en otros capitales, y que puede aumentar o disminuir, mejorando o empeorando las posibilidades de quien lo posea. Se fundamenta pues, en lazos permanentes y útiles, que se sostienen en intercambios, a la vez, materiales y simbólicos.

Precisando un poco más el concepto, es necesario tener en cuenta que lo que se "moviliza" no son estrictamente "personas", sino los capitales o recursos de los cuales están dotados esas personas: se ponen en marcha mecanismos que mueven poder, asociados a posiciones que ocupan agentes determinados, posiciones que tienen propiedades independientes de los individuos que las ocupan. Además, es también una fuente de poder, y por ello constituye "algo que está en juego" (enjeu), que se intenta acumular y por lo cual se está dispuesto a luchar.

\section{Las redes sociales en las estrategias de reproducción en la pobreza}

Desde el clásico análisis de Larissa Lomnitz en una barriada mexicana (Lomnitz, 1978), numerosos estudios sobre estrategias de sobrevivencia en la pobreza han reconstruido "redes de intercambio recíproco de bienes y servicios" con las cuales los pobres hacen frente a una serie de necesidades cotidianas, mostrando así la importancia de los recursos sociales en el análisis de la reproducción social. En otras palabras, siendo sólo uno de los tipos de recursos utilizables, el capital social cobra importancia fundamental en la medida en que se trata de comprender y explicar un conjunto de prácticas que son implementadas por un grupo de familias que poseen un escaso volumen de capital económico y cultural, y que se ven enfrentadas a 
REDES- Revista hispana para el análisis de redes sociales Vol. 14,\#4, Junio 2008

http: // revista-redes. rediris.es

resolver todas las exigencias de su cotidianeidad. Ello se liga, además, a una cuestión teórica y metodológica que considero fundamental y que he desarrollado en detalle en otros lugares: la necesidad de analizar las diferentes prácticas que generan las familias pobres para vivir, a partir de "lo que tienen" y no de "lo que les falta", de sus recursos, más que de sus "necesidades básicas insatisfechas". (Gutiérrez 2000, 2004a, 2004b, 2005).

Subrayo entonces, que es precisamente esta asociación de la noción de capital social (o recursos sociales) con la de red, la que permite la construcción de herramientas analíticas claves para el análisis relacional de la pobreza.

En efecto, sobre la base de estudios empíricos propios y de otros colegas, sostengo que en el sistema de las estrategias de reproducción social, las familias pobres generan prácticas - sin ser necesariamente conscientes de los mecanismos que permiten explicarlas y comprenderlas- que toman como apuesta principal su disponibilidad de capital social, movilizado en intercambios a través de diferentes tipos de redes, que pueden incidir, tanto en la superación como en la reproducción de sus condiciones de pobreza.

Así, dicho capital social puede cobrar diferentes formas (individual, familiar o doméstico y colectivo-comunitario), cada una de las cuales podrá dar lugar a la conformación de esas diferentes redes. Las redes pueden ser simétricas y asimétricas, y pueden consistir en intercambios de diferentes formas de capital (los otros recursos que se poseen, económicos, culturales, simbólicos) -que en términos de Mauss pueden llamarse como de "prestación total", en el sentido en que constituyen un conjunto complejo de reciprocidad indirecta, donde quien recibe la prestación no está directamente obligado a quien la ofrece sino a cualquier otro miembro del sistema- y fundamenta estrategias tanto individuales como colectivas.

El estado del arte respecto de esta cuestión presenta al menos cuatro construcciones típicas:

1. Red de intercambio de reciprocidad indirecta especializada: son redes que se instituyen entre pobres y no-pobres, quienes intercambian bienes y servicios asociados a distintas especies de capital, en las que las familias pobres apuestan su capital social colectivo y los no-pobres (agentes o instituciones) aportan otras especies de capital. (Gutiérrez, 2004a) 
REDES- Revista hispana para el análisis de redes sociales

Vol. 14,\#4, Junio 2008

http: // revista-redes. rediris.es

2. Red de intercambio diferido intergeneracional: son redes familiares entre familias pobres, que comprometen a la madre como principal productora y/o distribuidora y a los hijos e hijas y sus familias como principales receptores de diferentes circuitos de bienes y de servicios (Gutiérrez, 2004a).

3. Redes de resolución de problemas: son redes en las que circulan recursos en el marco de políticas sociales que compiten o se articulan con redes clientelares. (Auyero, 2001).

4. Redes de reciprocidad generalizada: son redes que sustentan parte de las prácticas que llevan a cabo los nuevos pobres para asegurarse recursos de parientes, amigos o conocidos. En ellas son importantes no solamente los recursos y servicios, sino también los significados y los juicios atribuidos. (Kessler, 1998).

\section{Un ejemplo de red de intercambio de reciprocidad indirecta especializada: con "Montoneros"}

Tomando especialmente la dimensión colectiva-comunitaria del capital social, y a partir del análisis de distintos aspectos ligados a la apropiación de la tierra, de la gestión del hábitat, y de la implementación de estrategias colectivas de un grupo de familias residentes en un barrio pobre cordobés, he podido mostrar cómo ciertas redes permiten el despliegue de determinadas estrategias de reproducción y generan escenas sociales donde se entrelazan sucesivamente modos de reproducción diferentes, que ligan a pobres y no-pobres en el marco de condiciones estructurales históricamente definidas.

Voy a centrarme aquí en una de esas redes: la que articuló a las familias estudiadas con un grupo de militantes Montoneros en la década de $1970^{5}$.

En efecto, originarios de dos villas miseria de la ciudad de Córdoba (Argentina) que constantemente sufrían las inundaciones del río que atraviesa el centro urbano, y ante una gran inundación producida en diciembre de 1972, un grupo de 29 familias que se vieron obligados a abandonar sus precarias viviendas- comienzan a desarrollar, junto a otros agentes sociales que ocupan otras posiciones en el espacio,

\footnotetext{
5 Para la reconstrucción de esa red, apelé a diferentes fuentes de información: bibliografía sobre Montoneros, documentos, libros testimoniales, y un conjunto de entrevistas que incluían a militantes disidentes, militantes que habían permanecido en la Organización y pobladores del barrio estudiado que habían ocupado diferentes posiciones en dicha red.
} 
REDES- Revista hispana para el análisis de redes sociales

Vol. 14,\#4, Junio 2008

http: // revista-redes. rediris.es

una serie de estrategias para conseguir y consolidar la apropiación de un nuevo hábitat en un terreno más seguro, alejado de la persistente amenaza del río Suquía ${ }^{6}$.

En esos tiempos, y en lo que se refiere específicamente a la conquista de la tierra que habitan actualmente, sus estrategias se entrelazan con las estrategias desplegadas por un grupo de Montoneros ${ }^{7}$ que ya venía actuando políticamente en las "villas de emergencia" de origen, en un período como el de 1970-1973, cuando el Movimiento se encuentra en un proceso de acumulación de capital político. Dicho proceso se caracterizaba por el fortalecimiento de los vínculos con las otras formaciones políticas peronistas, con un objetivo inmediato como grupo guerrillero urbano: el retorno del General Perón al poder como medio para llegar al socialismo en Argentina. Para ello, dentro de un marco definido como de "guerra integral", los militantes desarrollaban una serie de estrategias en villas y barrios pobres de la ciudad que consistían especialmente en la "organización activa de la gente", en su "concientización" y en obtener una suerte de "servicio logístico" para las acciones que llevaban a cabo.

Nosotros el trabajo de barrio que hacíamos era centralmente la organización de la gente, en general plantábamos un dispensario en todos lados, que era una de las primeras cosas que demandaba la gente y después resolver los problemas de terreno que no tenían, la construcción de viviendas y con el gobierno a estas cosas le fuimos dando respuesta oficial digamos (...) Era una organización participativa donde los dueños, digamos, para poner una expresión entre comillas, "dueños" del dispensario eran los vecinos y los médicos eran los que iban, los médicos y estudiantes de los últimos años. Para lo cual habíamos conseguido que se reconociera, que el trabajo de los estudiantes de Odontología o de Medicina que hacían en los barrios fuera

\footnotetext{
${ }^{6}$ En el momento de la inundación, las familias utilizaron, en un primer nivel, las redes formadas en sus antiguos asentamientos, sustentadas no sólo en la vecindad, sino también en fuertes lazos de parentesco, que constituyen, junto a las iguales condiciones objetivas, el fundamento de diferentes prácticas de intercambio de bienes y servicios de manera solidaria, unidos por relaciones que, en gran medida, pueden definirse como horizontales: la organización de los turnos para la comida, cuidar las pertenencias de manera colectiva, compartir los alimentos y las ropas que venían como donaciones de particulares o del Gobierno, trabajar en la búsqueda de una nueva tierra y en la gestión de su apropiación, etcétera, estrategias que continúan en los primeros tiempos de ocupación del terreno.

${ }^{7}$ El grupo "Montoneros" comienza a conformarse a fines de la década de 1960 en Argentina y aglutina fuerzas políticas que se desplazan progresivamente desde la "derecha" hacia la "izquierda". Constituyó la agrupación guerrillera urbana más importante de Latinoamérica, tanto por la cantidad de militantes que integraron sus filas, cuanto por el número de simpatizantes que convocó y por la cantidad y efectividad de las acciones que llevó a cabo, hasta su desmembramiento y aniquilación, especialmente durante la Dictadura Militar de 1976-1983 (Gillespi, 1987).
} 


\section{REDES- Revista hispana para el análisis de redes sociales Vol. 14,\#4, Junio 2008 \\ http: // revista-redes. rediris.es}

reconocido como parte de su formación académica (...) Particularmente con los de Odontología que debían hacer las prácticas en cualquier lugar. Venían y hacían en nuestros dispensarios (...) también parteras, de la Escuela de Enfermería" (Elbio, militante montonero).

Los "villeros", los "pobres", tienen en sí mismos, para los Montoneros, el valor de capital político colectivo: "pobre" es el "pueblo", fundamento de la legitimidad política y de la alianza, alianza posible por la existencia de "líderes" que la facilitan. Los "villeros pobres" tienen valor como apoyo político potencial, como manifestantes, como militantes, como electores potenciales: como "ciudadanos" en un sistema ideológico "democrático", que, además, son "ciudadanos pobres" a los que se puede ayudar, acompañar, para ligarse a ellos y establecer intercambios, y que simbolizan al "Pueblo", como "ciudadanos numerosos" y como "ciudadanos que tienen líderes" a través de los cuales se puede comprometer y fundamentar el intercambio.

Los ayudaron con cosa', así los ayudaron a trae', ¿viste? como ser asaltaban camione' ¿viste? de cometible' nos traían y los repartían, los tiraban así y hay muchos que no querían agarrá' nada, tenían miedo (...) Pero y si no, ayuda aparte d' eso era gente que los acompañaban, vamo' a poné' así ¿viste?. Acompañamiento de gente... mmmm no se qué podé' decí' para no decí montonero' (Rolo, líder de Villa Cañita).

Así, entre dos tipos de redes (la red política de Montoneros y la red local de los vecinos "pobres") se conforma una nueva red de intercambios en la cual puede reconstruirse un determinado sistema de dones y contra-dones, llamado de reciprocidad indirecta especializada. Por un lado, la red de Montoneros, como instrumento de reproducción, proporciona lo que los vecinos llaman "ayuda", capital económico bajo la forma de bienes materiales ${ }^{8}$. También proporciona fundamentalmente dos tipos de servicios que los vecinos Ilaman "acompañamiento": uno que es "acompañamiento" físico en la ocupación del nuevo terreno, fundado en un capital político clandestino acumulado y en una coyuntura política especial (el gobierno militar en la provincia de Córdoba de alguna manera permite la realización de las acciones), otro que es "acompañamiento" en las gestiones administrativas y

\footnotetext{
${ }^{8}$ En ese contexto, la "ayuda" se materializaba en diferente tipo de bienes: carne, yerba, aceite, azúcar y otros comestibles, guardapolvos, zapatillas, zapatos, colchones, remedios, etcétera. La comida era trasladada clandestinamente hasta la puerta de la villa y ahí se repartía con rapidez entre las familias, que acercaban todo tipo de recipientes para compartir lo que se traía, en especial en los primeros tiempos de la ocupación del terreno, cuando estaban levantando sus viviendas precarias.
} 
REDES- Revista hispana para el análisis de redes sociales Vol. 14,\#4, Junio 2008

http: // revista-redes. rediris.es

técnicas (marcado de los lotes en el nuevo terreno, selección de los lugares para construir las viviendas, etc.) que apuntan al proceso de obtención y de ocupación de la tierra, fundado en un primer momento en ciertos lazos con el gobierno militar y que luego de las elecciones democráticas cobra la forma de capital político oficial (por los fuertes lazos que une a Montoneros con el gobierno provincial elegido) y en un "saber hacer" universitario, un capital cultural acumulado por los militantes.

La red local de los vecinos ofrece como contra-don servicios políticos, fundados en un capital político colectivo que los lleva a participar en el proceso electoral institucional (manifestaciones, reuniones políticas, votos) y en actos políticos-simbólicos fundamentales en la época, capital político que encuentra su origen en un capital social colectivo, acumulación de relaciones de parentesco, vecindad y amistad que se había producido en las dos "villas miseria" de origen de los pobladores ${ }^{9}$.

Ayuda y acompañamiento a cambio de "ir a donde había que ir", bienes y servicios a cambio de servicios, definían el sistema de dones y contra-dones -entendido como la lógica social de invertir para intercambiar formas de capital- que enlazaba a los habitantes de la villa con el grupo de militantes montoneros en una red de intercambios recíprocos que tenía tres posiciones diferenciales dentro del conjunto de Ios participantes: Rolo y Cristóbal, por el lado de los pobladores del barrio, el Gordo Pipi, por el lado de Montoneros.

Esas posiciones eran estructuralmente homólogas en el doble sentido de dominantes y de dominados: Rolo y Cristóbal constituidos como "líderes naturales" en las villas que habitaban a orillas del Suquía, fundan su dominación en la red local que se conforma en el nuevo asentamiento; el Gordo Pipi, dominante en la red de Montoneros en sus enlaces con las villas y barrios pobres del este de la ciudad, dominación fundada en su condición de "líder natural" de la zona. Dominados, considerando espacios de juego más amplios: el Gordo Pipi, según el testimonio de militantes montoneros, "no era un jefe en la organización", era un militante encuadrado en la seccional 5a que, siendo parte del "pueblo", de las "bases" (era un "militante de base, bien de base") actuaba de nexo con el "pueblo" pero no participaba de las grandes tomas de decisiones del movimiento; Cristóbal y Rolo

${ }^{9}$ Otros servicios incluían la "atención" de los militantes cuando organizaban una reunión del movimiento que concentraba a personas que venían desde distintos puntos del país: ello incluía la preparación de la comida, servir a la gente, lavar los platos y otros enseres domésticos, cebar mate, limpiar el lugar de la reunión y estar atento a lo que necesitaran. Este servicio era realizado por mujeres, que no recibían dinero por ello, y que sentían que "devolvían la ayuda que recibían". 


\section{REDES- Revista hispana para el análisis de redes sociales Vol. 14,\#4, Junio 2008 \\ http: // revista-redes. rediris.es}

(como el resto de sus vecinos) eran "el pueblo", los dominados del espacio social global, parte "necesaria" en el movimiento que aspiraba a la instauración del socialismo.

Es importante destacar que, a modo de balance y tras esa red de intercambios de reciprocidad indirecta especializada que se sostiene durante aproximadamente cuatro años, los vecinos obtuvieron fundamentalmente dos cosas. En primer lugar, una acumulación de capital económico bajo la forma de tierra, es decir, de un nuevo espacio físico en un terreno del cual inician un camino de apropiación, a partir de una cesión del Gobierno Provincial, tras la reconversión del capital social que habían logrado acumular. En segundo lugar, un reforzamiento de ese capital social previamente acumulado, resultado de un efecto de estructuración sobre el espacio siendo aquí el espacio una relación social dentro de un territorio-, sostenido en dos cuestiones: por un lado, en el reconocimiento de Rolo y de Cristóbal, los dos "líderes naturales" (uno de cada una de las "villas miseria" de origen), como efecto de una diferenciación de los "conscientes", de una diferenciación de la diferencia, de la identificación de un capital político fundamentado en una forma social, aunque se apele a la naturaleza (son "líderes naturales") para dar a los políticos-intelectuales la base de su legitimidad. Por otro lado, en la unificación de las dos redes sociales (una de cada una de las villas miseria de origen) que implica la creación de un capital social colectivo, reconvertible en un capital político de mayor fuerza, que constituye el fundamento de los servicios políticos. Además, este capital social colectivo facilita la movilización del capital político de los Montoneros para apoyar las gestiones administrativas de la cesión del terreno.

El regreso del Movimiento Montoneros a la clandestinidad y el comienzo de las acciones represivas contra sus militantes (que comienzan en el año 1974), constituyen las características más sobresalientes del cambio de las condiciones estructurales que hacen que el capital social acumulado deje de reconvertirse en capital político o, en otras palabras, que el capital político que se había acumulado alcance un alto punto de desvalorización, y lleva a los pobladores del nuevo asentamiento a acumular desconfianza en la reconversión del capital social en capital político colectivo, y con ello, a la descomposición de la red de intercambios que los había unido al Movimiento, hasta desaparecer por completo con el Golpe de Estado de 1976. En realidad, la red consistía en una suerte de juego clientelar, donde los vecinos dicen que tomaban a los militantes montoneros "como políticos" -en el 
REDES- Revista hispana para el análisis de redes sociales

Vol. 14,\#4, Junio 2008

http: // revista-redes. rediris.es

sentido de político-partidario-, es decir como recursos que pueden ser movilizados para obtener otro tipo de bienes, pero que, llegado ese momento especial de la dinámica estructural, comienza a perder su valor de reconversión en otros tipos de capitales.

Como signo de los nuevos tiempos que corrían, la "villa" asentada en el nuevo terreno comienza a llamarse "Villa Altos de San Martín", ocultando el sentido revolucionario de su antiguo nombre, "Villa 29 de mayo"10.

Bueno ellos lo pusieron, Y el Gordo Pipi. Ellos lo' ayudaron a nosotro' a poné' el nombre. 'Tonce' nosotro' como éramo' ignorante' dijimo' "bueno, que sea el 29 de mayo". Pero no lo' acordábamo' que era del Cordobazo. No que ahí nomá' de la Casa 'e Gobierno lo baja, lo sacaron del nombre, usted no pueden llevá ese nombre. (...) Fue una vez que fuimo' a hacé' unos trámite'. 'Ustede' acá no pueden figurá' con ese nombre", dice. "Póngalen 23, 22", me acuerdo que lo retaban "pero ustede' no pueden llevá esa fecha" dice. (...) Y bueno, le pusimo' Alto San Martín" (Lola).

El mismo sistema de intercambio de dones y contra-dones basados en diferentes especies de capital (y en el caso de las familias pobres, en su capital social colectivo), puede ser visualizado en otras circunstancias estructurales y con otros agentes sociales que ocupan -como los Montoneros- otras posiciones en el espacio social. He reconstruido una red similar que entrelazó a las familias del barrio estudiado con dos ONGs en un prolongado proceso de gestión y consolidación del hábitat y de la institucionalización de su capital social colectivo como Cooperativa de Vivienda y Consumo, y otras redes que las ligan a agentes políticos-partidarios en los momentos pre-electorales, con los cuales intercambian favores políticos (capital social colectivo transformado en capital político) por distintas formas de capital económico (dinero para el comedor y otros emprendimientos colectivos, alimentos, promesa de algún trabajo, etc.).

\footnotetext{
${ }^{10}$ El 29 de mayo de 1969 se desencadenó el Ilamado “Cordobazo", una fusión de protesta estudiantil y descontento obrero que envolvió a la segunda ciudad del país en luchas callejeras, en medio de una huelga general de acatamiento masivo. Terminó en una cruenta intervención de las fuerzas armadas, en la que hubo catorce muertos. La impresionante movilización de columnas de estudiantes y obreros que estratégicamente se van concentrando hacia el centro de la ciudad, el alto contenido contestatario de la protesta frente a un gobierno militar que se quebraba y la fuerte participación popular en el movimiento son imágenes que evoca la fecha "29 de mayo", fecha que no remite directamente a montoneros (la organización que existía en ese momento no participó como tal) sino que luego será reivindicada por todos los grupos revolucionarios de la época.
} 
REDES- Revista hispana para el análisis de redes sociales

Vol. 14,\#4, Junio 2008

http: // revista-redes. rediris.es

Así, la constitución y el mantenimiento de diferentes redes de intercambio de reciprocidad indirecta especializada, permiten la construcción de escenas sociales que articulan sucesivamente varios modos de reproducción diferentes: básicamente el de los pobres en la pobreza y los de los no-pobres en sus campos específicos: el de los montoneros en el campo político nacional y local, el de las ONGs en su propio espacio de juego en disputa por "beneficiarios", el de los políticos-partidarios en su lucha por captar "clientela". Incluso, con el correr del tiempo, cuando ese capital social colectivo es monopolizado por un número reducido de familias (las que, en primera línea, ocupan los cargos directivos de la Cooperativa del barrio y las que, en segunda línea, administran y gestionan los recursos colectivos), se articulan dos modos específicos de reproducción de los pobres: el de los monopolistas y el del resto de las familias que sólo actúan como "público" o "beneficiarios"11.

\section{A modo de cierre: el análisis de redes en sus dimensiones estructural y vincular}

Puede verse entonces que, especialmente en la conformación de redes que unen a pobres con no-pobres, hay que tener en cuenta que una de las formas fundamentales del capital social es el colectivo, y, más concretamente, sus posibilidades de reconversión en otras especies de capital (capital político, capital militante -Matonti y Poupeau 2005-, etc.), formas reconvertidas que no pueden definirse a priori, sino que están en relación con condiciones estructurales históricamente situadas, $y$, por ello, observables en problemáticas empíricas concretas.

La noción de capital social se muestra entonces como una herramienta fecunda para analizar ciertas dimensiones de las estrategias de reproducción social. A partir de los recursos que tienen, quienes viven en situaciones de pobreza despliegan una serie de estrategias que se entrelazan con las estrategias de otros agentes o grupos sociales que tienen sus apuestas en otros juegos. Conforman así cierto tipo de redes sociales que, de alguna manera, constituyen espacios de articulación de uno y otro modo de reproducirse socialmente. El análisis de dichas redes permite iluminar una

\footnotetext{
${ }^{11}$ Pensar en capital social colectivo supone también sostener la hipótesis de la existencia de una lucha por su apropiación (o monopolización) entre los distintos componentes de las redes, lo que implica tener presente la existencia de tensiones y de conflictos, y, con ello, la estructuración y reestructuración de relaciones de poder, no sólo entre pobres y no-pobres, sino también entre quienes viven y comparten situaciones de pobreza.
} 
REDES- Revista hispana para el análisis de redes sociales

Vol. 14,\#4, Junio 2008

http: // revista-redes. rediris.es

dimensión importante de la reproducción social en su conjunto y de sus mecanismos de dominación.

En efecto, sostengo que para avanzar en la comprensión y explicación del fenómeno de la pobreza y de los mecanismos que la sustentan y perpetúan, más que resolver si los pobres se encuentran o no al margen del espacio social, si están excluidos o no, es importante develar la manera como se sitúan en ese espacio, a partir de qué capitales (objetivados e incorporados), en relación con cuáles instrumentos de reproducción y en función de qué estado de la relación de fuerzas entre las clases.

En ese marco, propongo una doble dimensión para el análisis de las redes sociales:

1) Una dimensión estructural, que remite a las condiciones objetivas externas (históricamente situadas) y a su relación con aquellos elementos que permiten ubicar a los agentes e instituciones en el espacio social: volumen y estructura del capital (objetivado e incorporado) ${ }^{12}$. Me refiero al capital social, evidentemente, pero también a las otras formas de capital (económico, cultural y simbólico) que se intercambian: esas diferentes especies de capital constituyen así las condiciones de posibilidad de la circulación de distintos tipos de bienes y servicios entre los componentes de las redes. Para la construcción de esta dimensión estructural, el análisis de correspondencias múltiples se muestra especialmente adecuado. En efecto, este método hace posible la representación del espacio social considerado a través de planos factoriales que, a modo de diagramas, posibilitan el análisis y visualización del conjunto de relaciones que se presentan simultáneamente entre las propiedades observadas. Esto es, el sistema completo de relaciones que constituye el verdadero principio de fuerzas que actúan en ese espacio. Así, en estos planos es posible representar y ubicar cada modalidad interviniente de cada variable considerada (en este caso, variables que remiten a las distintas especies de capital - económico, cultural, social y simbólico- con que cuentan las familias y los otros agentes involucrados) y analizar su significado conforme a su posición en el sistema de diferencias construido. Del mismo modo, esta representación del espacio social permite proyectar y visualizar la ubicación relativa de los agentes presentes en él (en este caso, agentes individuales, pero también colectivos, como la familia considerada como

${ }^{12}$ Considerar dialécticamente las formas objetivadas e incorporadas de los capitales, constituye otra de las dimensiones del análisis relacional que propongo. 
REDES- Revista hispana para el análisis de redes sociales Vol. 14,\#4, Junio 2008

http: // revista-redes. rediris.es

unidad doméstica). Por último, y a partir de las proximidades y distancias en este espacio, es posible el armado de clases construidas sobre la base de las propiedades que devienen de la posición de cada agente en la estructura del sistema de relaciones. Estas clases construídas permiten identificar, por ejemplo, "cuadros de disponibilidad de recursos" diferentes, es decir, estructuras patrimoniales que suponen distintos tipos de recursos económicos, culturales, sociales y simbólicos y distinta cantidad de cada uno de ellos. Al ser diferentes, esos cuadros de disponibilidad de recursos condicionan estructuralmente de modo diferente a las unidades familiares, a adoptar distintas estrategias de reproducción social y a estar en mayor o menor medida "dispuestas" (como disposición objetiva e incorporada) a conformar redes entre sí o con otros agentes e instituciones del espacio.

2) Una dimensión vincular, que consiste en el análisis de las interacciones concretas, de su frecuencia, de la intensidad de los lazos que pudieran crearse, del tipo y calidad de los bienes (materiales o no-materiales) y de los servicios que circulan, etc. Aquí es importante incluir en el análisis elementos que hacen a la subjetividad de los agentes implicados, ligados a trayectorias individuales y a experiencias personales: con ello aludo al segundo nivel de lo "relacional" del capital social del que habla Baranger (op. cit.), y que he mencionado más arriba. Esta estructura de vínculos puede ser construida y visualizada a partir de Ucinet o de programas semejantes. Pero, más allá de ello, el abordaje de la dimensión histórica y de los aspectos simbólicos de la problemática considerada, lleva a apelar a un despliegue importante de metodologías cualitativas, que incluyen observación de las prácticas concretas, entrevistas e historias de vida, éstas últimas consideradas, no como biografías sino como trayectorias: es decir, como reconstrucción relacional de las posiciones sucesivas que han ocupado por referencia al espacio de posiciones considerado, las unidades de análisis implicadas, sean éstos agentes individuales o colectivos (familias, instituciones, etc.). 
REDES- Revista hispana para el análisis de redes sociales Vol. 14,\#4, Junio 2008

http: // revista-redes. rediris.es

En definitiva, quiero subrayar que estos dos conceptos relacionales como el de red y el de capital social, lo son en la medida en que aluden a relaciones (vínculos) que se establecen entre agentes e instituciones, pero también, lo son en el otro sentido que he mencionado a lo largo de este trabajo: el de las estructuras objetivas (materiales y simbólicas, externas e incorporadas), que son independientes de esos agentes e instituciones y que constituyen los límites y las potencialidades de esos vínculos.

Utilizar ambos conceptos como herramientas empíricas para el análisis de las estrategias de reproducción social en la pobreza y de su articulación con la reproducción de la sociedad en su conjunto, supone pues, tener en cuenta las dos dimensiones de las redes, la estructural y la vincular, y las consecuencias teóricas y metodológicas que ello conlleva.

\section{Bibliografía}

Auyero, Javier (2001). La política de los pobres. Las prácticas clientelísticas del peronismo. Buenos Aires: Manantial.

Baranger, Denis (2000). "Sobre estructuras y capitales: Bourdieu, el análisis de redes, y la noción de capital social", en: Avá, no 2, pp. 41-63.

Bourdieu, Pierre (1980). "Le capital social. Notes provisoires", en: Actes de la Recherche en Sciences Sociales, no 31, pp. 2-3. [El capital social. Notas provisorias. En Bourdieu, Pierre. Campo del poder y reproducción social. Elementos para un análisis de la dinámica de las clases, Córdoba: Ferreyra Editor.]

Coleman, James (1987). “Norms as Social Capital". En: Radnitzky, G. y Bernholz, P. (Eds.). Economic Imperialism: The Economic Method Applied outside the Field of Economics. New York: Paragon House Publishers.

- (1988). "Social Capital in the Creation of Human Capital", en: American Journal of Sociology, 94, pp. 95-121.

- (1990). Foundations of Social Theory. Cambridge: Harvard University Press.

Gillespie, Richard (1987). Soldados de Perón. Los montoneros. Buenos Aires: Grijalbo.

Gutiérrez, Alicia (2000). “Reflexiones teórico-metodológicas en torno al análisis de la pobreza". En: Rodríguez M. y Rozé, J. (Comp.). Ciudades Latinoamericanas: Una Visión Social del Urbanismo. (pp. 91-106). Guerrero: Fomento UniversitasUniversidad Autónoma de Guerrero.

- (2004a). Pobre', como siempre.... Estrategias de reproducción social en la pobreza. Córdoba: Ferreyra Editor.

- (2004b). “La teoría de Bourdieu en la explicación y comprensión del fenómeno de la pobreza urbana". En: Martín Criado, E. Alonso, L. y Moreno Pestaña, J. (Comp.). Pierre Bourdieu: las herramientas del sociólogo. (pp. 255-280). Fundamentos: Madrid. 
REDES- Revista hispana para el análisis de redes sociales Vol. 14,\#4, Junio 2008

http: // revista-redes. rediris.es

-(2005). “Acerca de la noción de capital social como herramienta de análisis. reflexiones teóricas en torno a un caso empírico", en: Perspectivas, no 2, Centro de Estudios en Administración, Políticas Públicas y el Estado, Universidad Nacional del Comahue, pp. 7-26.

-(2006). "Clases, espacio social y estrategias: una introducción al análisis de la reproducción social en Bourdieu". En: Bourdieu, P., Campo del poder y reproducción social. Elementos para un análisis de la dinámica de las clases. (pp. 9-27). Córdoba: Ferreyra Editor.

-(2007), “El 'Capital social' en la pobreza: apuesta, medio y resultado de luchas simbólicas". En: Pavcovich, P. y Truccone, D. (Comp.). Aproximaciones teóricas al estudio de la pobreza en Argentina, Villa María: Ed. de la Universidad Nacional de Villa María. (En prensa).

Hintze, Susana (2004). "Capital social y estrategias de supervivencia. Reflexiones sobre el "capital social de los pobres'". En: Danani, C. (Comp.). Política social y economía social. Debates fundamentales. (pp. 143-166). Buenos Aires: AltamiraFundación OSDE-UNGS.

Kessler, Gabriel (1998). "Lazo social, don y principios de justicia: sobre el uso del capital social en sectores medios empobrecidos". En de Ipola, E. (Comp.). La crisis del lazo social, Durkheim cien años después. Buenos Aires: Eudeba.

Lomnitz, Larissa (1978). Cómo sobreviven los marginados. México: Siglo XXI.

Matonti, Fréderic y Poupeau, Franck (2005). "Le capital militant. Essai de définition", en : Actes de la recherche en sciences sociales, no 155, pp. 5-11.

Millán, R. y Gordon, S. (2004). "Capital social: una lectura de tres perspectivas clásicas", en: Revista Mexicana de Sociología, año 66, no 4, pp. 711-747.

Putnam, Robert (1993). Making Democracy Work: Civic Traditions in Modern Italy. Princeton, N.J .: Princeton University Press.

-(1995). “Tuning In, Tuning Out: The Strange Disappearance of Social Capital in America", en: Political Science and Politics (diciembre), pp. 664-683.

Woolcock, M. y Narayan, D. (2000). Social capital: Implications for Development Theory, Research and Policy, en: The World Bank Observer, 15, pp. 225-249. 